\title{
Birack shadow modules and their link invariants
}

\author{
Sam Nelson* $\quad$ Katie Pelland ${ }^{\dagger}$
}

\begin{abstract}
We introduce an associative algebra $\mathbb{Z}[X, S]$ associated to a birack shadow and define enhancements of the birack counting invariant for classical knots and links via representations of $\mathbb{Z}[X, S]$ known as shadow modules. We provide examples which demonstrate that the shadow module enhanced invariants are not determined by the Alexander polynomial or the unenhanced birack counting invariants.
\end{abstract}

KEYWORDS: biracks, birack shadows, shadow algebra, shadow modules, link invariants, enhancements of counting invariants

2010 MSC: 57M27, 57M25

\section{Introduction}

In [1] an associative algebra was introduced arising from a finite quandle $X$, known as the quandle algebra $\mathbb{Z}[X]$, with representations known as quandle modules. In [5] and later [10], quandle modules and rack modules were used to define enhancements of the quandle and rack counting invariants.

In [3] the rack algebra was generalized to the case of finite biracks. In this paper we generalize the birack algebra further to the case of birack shadows, pairs $X, S$ where $X$ is a birack and $S$ is a set with an action of $X$ satisfying certain properties. The shadow algebra of a birack shadow $X, S$ and its representations, known as shadow modules, are used to further enhance the birack shadow counting invariant for classical knots and links.

The paper is organized as follows. In section 2 we recall the basics of biracks and birack shadows. In section 3 we introduce the shadow algebra and shadow modules. In section 4 we define the shadow module enhanced counting invariant and compute some examples, including examples which show that the invariant is strictly stronger than the unenhanced birack shadow counting invariant and is not determined by the Alexander polynomial. We conclude with a few questions for future research in section 5

\section{Biracks and Shadows}

We begin by recalling a definition (see [9, 7] or [15, for more).

Definition 1 Let $X$ be a set. A birack structure on $X$ is an invertible map $B: X \times X \rightarrow X \times X$ satisfying the conditions

(i) $B$ is sideways invertible, that is, there exists a unique map $S: X \times X \rightarrow X \times X$ satisfying for all $x, y \in X$

$$
S\left(B_{1}(x, y), x\right)=\left(B_{2}(x, y), y\right),
$$

\footnotetext{
*Email: knots@esotericka.org

†Email: katie.pelland@gmail.com
} 
(ii) $B$ is diagonally invertible, that is, the components $\left(S^{ \pm 1} \circ \Delta\right)_{1}$ and $\left(S^{ \pm 1} \circ \Delta\right)_{2}$ of the compositions $S \circ \Delta$ and $S^{-1} \circ \Delta$ of the diagonal map $\Delta(x)=(x, x)$ with sideways map and its inverse are bijections, and

(iii) $B$ is a solution to the set-theoretic Yang-Baxter equation:

$$
(B \times I)(I \times B)(B \times I)=(I \times B)(B \times I)(I \times B) .
$$

We will occasionally find it convenient to abbreviate $B_{1}(x, y)=y^{x}$ and $B_{2}(x, y)=x_{y}$.

We interpret the birack operation $B(x, y)$ as taking labels $x, y \in X$ on the input semiarcs at a positive crossing and assigning labels to the output semiarcs. The inverse map $B^{-1}$ represents going through a negative crossing, and the sideways map represents going through a positive crossing sideways from left to right.
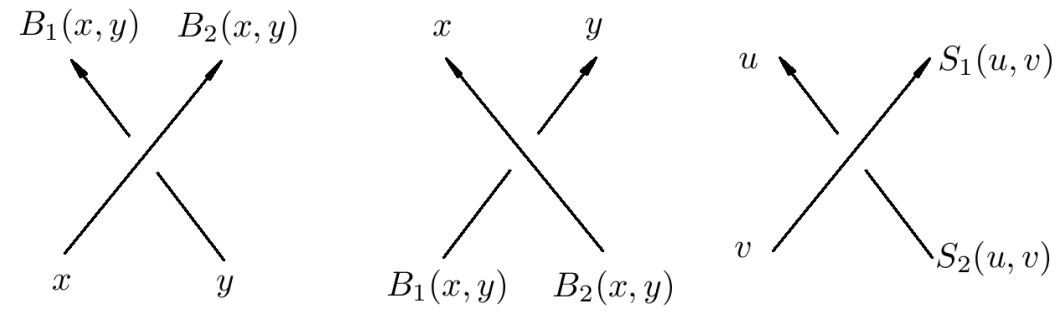

The birack axioms are chosen such that labelings of the semiarcs in an oriented blackboardframed knot or link diagram are preserved by blackboard-framed isotopy moves. Invertibility and sideways invertibility satisfy the direct and reverse type II moves, and the Yang-Baxter criterion satisfies the type III move. Diagonal invertiblity satisfies the framed type I moves.
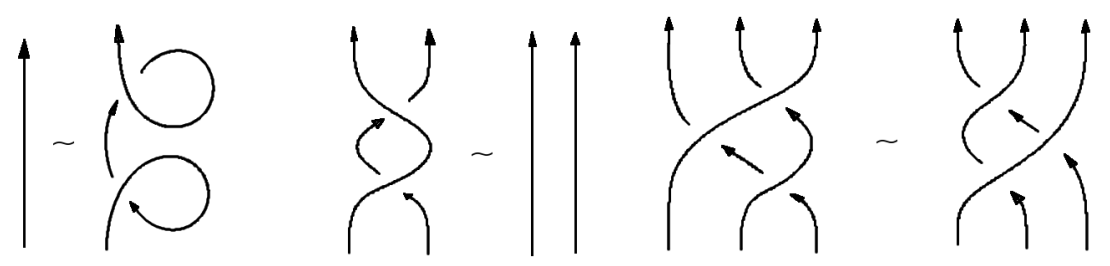

The bijections $\alpha: X \rightarrow X$ and $\pi: X \rightarrow X$ defined by $\alpha=\left(S^{-1} \circ \Delta\right)_{2}^{-1}$ and $\pi=\left(S^{-1} \circ \Delta\right)_{1} \circ \alpha$ determine the labels on the semiarcs introduced in a blackboard framed type I move.

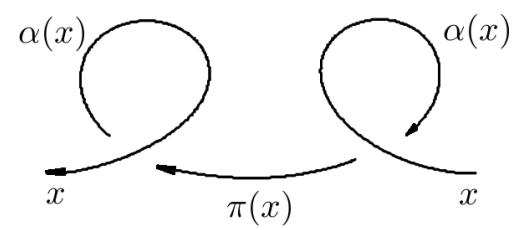

If $X$ is a finite birack, then $\pi$ is an element of the finite symmetric group $S_{|X|}$. The order or exponent of $\pi$, i.e. the smallest positive integer $N$ such that $\pi^{N}(x)=x$ for all $x \in X$, is known as the birack rank or birack characteristic of $X$. If $X$ is a birack of rank $N$, then birack labelings of a link $L$ by $X$ are preserved by the $N$-phone cord move: 


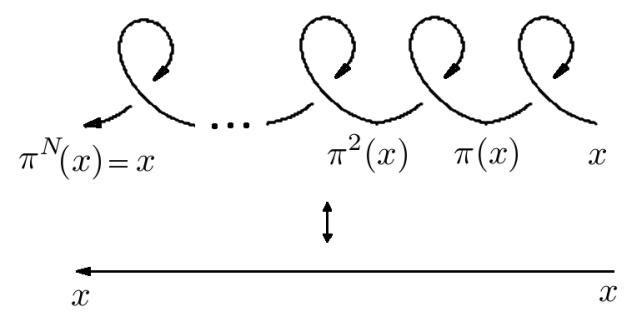

Examples of biracks include

- Groups. A group $G$ is a birack under the map $B(x, y)=\left(x^{n} y x^{-n}, x\right)$, for instance; many other birack structures on groups exist.

- Quandles and racks. A quandle $Q$ (see [11, 13]) or a rack $R$ (see [8]) is a birack under $B(x, y)=(y \triangleright x, x)$.

- Biquandles. A strong biquandle $X$ (see [12, 7]) is a birack under $B(x, y)=\left(y^{x}, x_{y}\right)$.

- Vector Spaces. A vector space $V$ over a field $\mathbb{F}$ is a birack under $B(x, y)=(t y+s x, r x)$ where $t, s, r \in \mathbb{F}$ satisfy $s^{2}=(1-t r) s$; biracks of this type are known as $(t, s, r)$-biracks. See [15].

If $X=\left\{x_{1}, \ldots, x_{n}\right\}$ is a finite birack, we can encode the birack structure with a block matrix $M_{X}=[U \mid L]$ where the $i, j$ entry of $U$ is $k$ where $x_{k}=B\left(x_{j}, x_{i}\right)$ and the $i, j$ entry of $L$ is $l$ where $x_{l}=B\left(x_{i}, x_{j}\right)$. Note the reversed order of $i, j$ in $U$; this is for compatibility with previous work.

We have the following standard notions:

Definition 2 Let $X$ and $X^{\prime}$ be sets with birack structures $B$ and $B^{\prime}$. Then we have:

- Homomorphisms. A map $f: X \rightarrow X^{\prime}$ is a birack homomorphism if for all $x, y \in X$ we have

$$
B^{\prime}(f(x), f(y))=\left(f\left(B_{1}(x, y)\right), f\left(B_{2}(x, y)\right)\right),
$$

that is, if we have $B^{\prime} \circ(f \times f)=(f \times f) \circ B$.

- Subbiracks. A subset $Y \subset X$ is a subbirack of $X$ if the restriction of $B$ to $Y \times Y$ defines a birack structure on $Y$; equivalently, $Y \subset X$ is a subbirack if $Y \times Y$ is closed under $B$.

Given a finite birack $B$ of rank $N$ and a blackboard framed oriented link diagram $L$ of $c$ components, the set of labelings of semiarcs in $L$ satisfying the crossing conditions

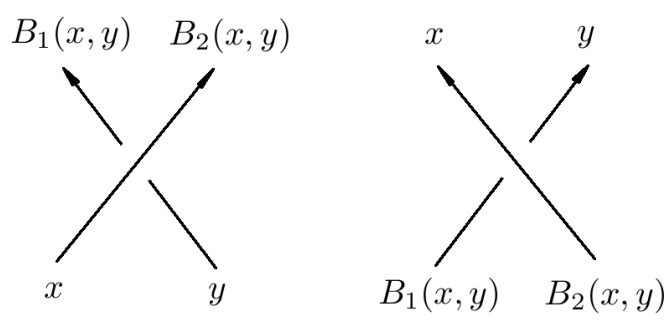

at every crossing corresponds bijectively with the set $\operatorname{Hom}(B R(L), B)$ of birack homomorphisms from the fundamental birack of $L$ (see [7, 15]) to $B$. Moreover, changing $L$ by blackboard framed moves and $N$-phone cord moves also yields a bijection on the sets of labelings; thus, summing over a 
complete period of framings modulo $N$ yields a link invariant known as the integral birack counting invariant

$$
\Phi_{B}^{\mathbb{Z}}(L)=\sum_{\mathbf{w} \in(\mathbb{Z})^{N}}|\operatorname{Hom}(B R(L, \mathbf{w}), B)|
$$

where $\mathbf{w}=\left(w_{1}, \ldots, w_{c}\right)$ is the vector of framing numbers of $L$. See [15] for more.

If the elements of a birack are used to label the semiarcs of a an oriented blackboard-framed classical link diagram on the sphere $S^{2}$, we can use elements of another set $S$ called shadows to label the regions between the arcs. We can then let the birack label on a semiarc act on the label in one region bounded by the semiarc to determine the label in the opposite region as pictured below.

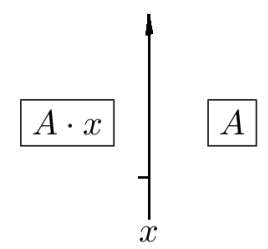

We want birack shadows labelings to be preserved by blackboard-framed Reidemeister moves. To guarantee this, we need the shadow labelings to be well-defined at crossings and at kinks.

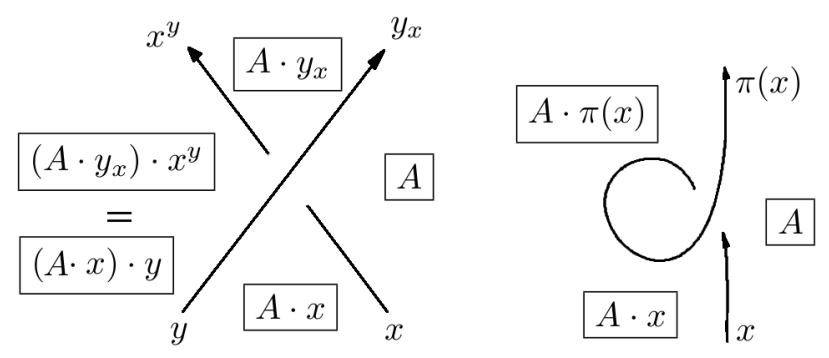

Thus we have:

Definition 3 Let $X$ be a birack and $S$ a set. A birack shadow structure on $S$ is an invertible right action of $X$ on $S$ (i.e. a map $\cdot: X \times S \rightarrow S$ ) satisfying for all $x, y \in X$ and $A \in S$,

(i) $\left(A \cdot y_{x}\right) \cdot x^{y}=(A \cdot x) \cdot y$ and

(ii) $A \cdot x=A \cdot \pi(x)$.

We will refer to such an $S$ as an $X$-shadow.

If $X=\left\{x_{1}, \ldots, x_{n}\right\}$ is a finite birack and $S=\left\{A_{1}, \ldots, A_{m}\right\}$ is a finite set, we can encode an $X$-shadow structure on $S$ with an $m \times n$ matrix $M_{X, S}$ whose $i, j$ entry is $k$ where $A_{k}=A_{i} \cdot x_{j}$.

Let $L$ be an oriented blackboard-framed link diagram on $S^{2}, X$ a finite birack and $S$ a finite $X$ shadow. A shadow labeling of $L$ is a labeling of the semiarcs of $L$ with elements of $X$ and the regions between the semiarcs of $L$ with elements of $S$ such that at every crossing and region boundary we have
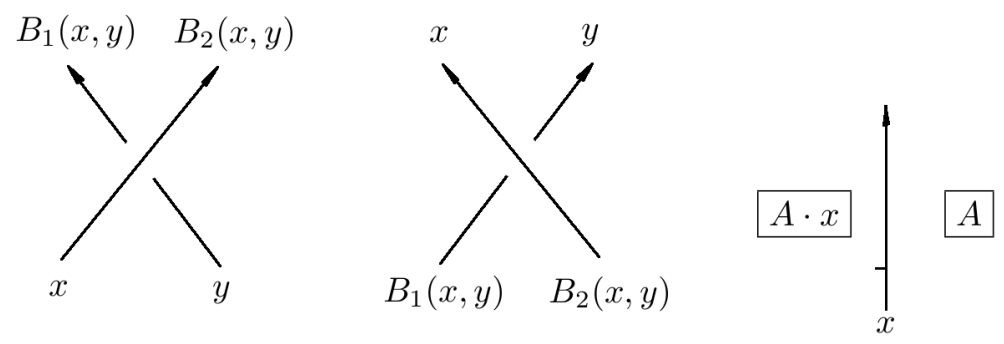
While it is true that the number of shadow labelings $\Phi_{X, S}^{\mathbb{Z}}(L)$ of a link $L$ by a birack-shadow pair $(X, S)$ over a complete period of writhes is a link invariant, unfortunately $\Phi_{X, S}^{\mathbb{Z}}(L)$ is determined by the usual birack counting invariant $\Phi_{X}^{\mathbb{Z}}(L)$, since for any birack labeling we can choose a shadow label for a starting region and simply push it across the semiarcs using the shadow operation and its inverse to determine a unique valid shadow labeling. More precisely, we have

Theorem 1 The number of shadow labelings of a link $L$ by $(X, S)$ is given by

$$
\Phi_{X, S}^{\mathbb{Z}}(L)=|S| \Phi_{X}^{\mathbb{Z}}(L) .
$$

Despite this fact, enhancements of the shadow counting invariant can give us information allowing us to distinguish links with equal birack counting invariant. Previously studied examples include quandle 3-cocycle invariants (where $X$ is a finite quandle, i.e. a rank $N=1$ birack with $B_{2}(x, y)=x$, $S=X$ and $A \cdot x=B_{1}(x, A)$, see [6]) and rack shadow polynomials (see [4]). For the present paper, we will generalize the rack module idea from [1] to define shadow modules, which we will use to enhance the birack shadow counting invariant.

Remark 1 If $L$ is a virtual link, the only shadow labelings which are preserved by virtual Reidemeister moves are constant labelings, i.e. shadow labelings such that $A \cdot x=A$ for all $A \in S, x \in X$. Unfortunately, this fact cannot be used to detect non-classicality, only non-planarity of a particular diagram of a virtual knot or link, since even classical knots and links have non-planar virtual diagrams.

\section{The Shadow Algebra and Shadow Modules}

In 11 an associative algebra is defined with generators corresponding to ordered pairs of elements of a finite quandle $X$, with relations obtained from Reidemeister moves with a labeling of the strands by elements of $X$. In [10 the quandle algebra was modified slightly for the purpose of enhancing the integral rack counting invariant described in [14. In [3] the rack algebra was generalized to the cae of biracks. We will now generalize one step further to the case of birack shadows.

Let $L$ be a classical knot or link diagram, $X$ a finite birack, $S$ an $X$-shadow and fix a shadow labeling of $L$ by $X, S$. We place 'beads' on the semiarcs of $L$ related by linear equations with coefficients depending on the rack and shadow labels at the crossings as depicted:
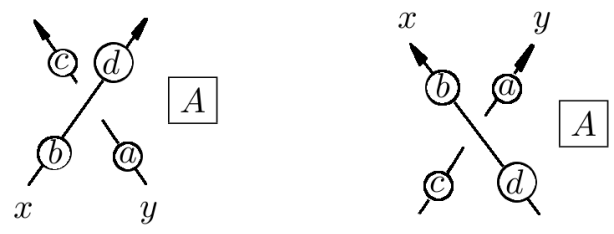

$$
\begin{aligned}
c & =t_{A, x, y} b+s_{A, x, y} a \\
d & =r_{A, x, y} a
\end{aligned}
$$

Definition 4 Let $X$ be a birack with birack rank $N$ and let $(S, \cdot)$ be a birack shadow. Let $\Omega[X, S]$ be the free $\mathbb{Z}$-algebra generated by elements of the form $t_{A, x, y}^{ \pm 1}, s_{A, x, y}$ and $r_{A, x, y}^{ \pm 1}$ for $A \in S, x, y \in X$. Then the shadow algebra $\mathbb{Z}[X, S]$ is the quotient of $\Omega[X, S]$ by the ideal $I$ generated by elements of the form

- $r_{A, x_{z} y, y_{z}} r_{A \cdot y_{z}, x, z^{y}}-r_{A, x_{y}, z} r_{A \cdot z, x, y}$

- $t_{A, x_{z} y, y_{z}} r_{A, y, z}-r_{A \cdot x_{y z}, y^{x}, z^{x y}} t_{A \cdot z, x, y}$,

- $s_{A, x_{z} y, y_{z}} r_{A \cdot y_{z}, x, z^{y}}-r_{A \cdot x_{y z}, y^{x}, z^{x y}} s_{A \cdot z, x, y}$,

- $t_{A \cdot y_{z}, x, z^{y}} t_{A, y, z}-t_{A \cdot x_{y z}, y^{x}, z^{x y}} t_{A, x_{y}, z}$, 
- $t_{A \cdot y_{z}, x, z^{y}} s_{A, y, z}-s_{A \cdot x_{y z}, y^{x}, z^{x y}} t_{A \cdot z, x, y}$,

- $s_{A \cdot y_{z}, x, z^{y}}-t_{A \cdot x_{y z}, y^{x}, z^{x} y} s_{A, x_{y}, z} r_{A \cdot z, x, y}-s_{A \cdot x_{y z}, y^{x}, z^{x_{y}}} s_{A \cdot z, x, y}$, and

- $1-\prod_{k=0}^{N-1}\left(t_{A \cdot-1} \alpha\left(\pi^{k}(x)\right), \pi^{k}(x), \alpha\left(\pi^{k}(x)\right) r_{A \cdot{ }^{-1} \alpha\left(\pi^{k}(x)\right), \pi^{k}(x), \alpha\left(\pi^{k}(x)\right)}+s_{A \cdot{ }^{-1} \alpha\left(\pi^{k}(x)\right), \pi^{k}(x), \alpha\left(\pi^{k}(x)\right)}\right)$.

A shadow module or $(X, S)$-module is a representation of $\mathbb{Z}[X, S]$, i.e. an abelian group $G$ with a family of automorphisms $t_{A, x, y}, r_{A, x, y}: G \rightarrow G$ and endomorphisms $s_{A, x, y}: G \rightarrow G$ such that each of the above maps is zero for all $A \in S$ and $x, y \in X$.

The conditions in definition 4 come from comparing the beads on the two sides of the oriented framed Reidemeister moves and the $N$-phone cord move using the labeling convention defined above.
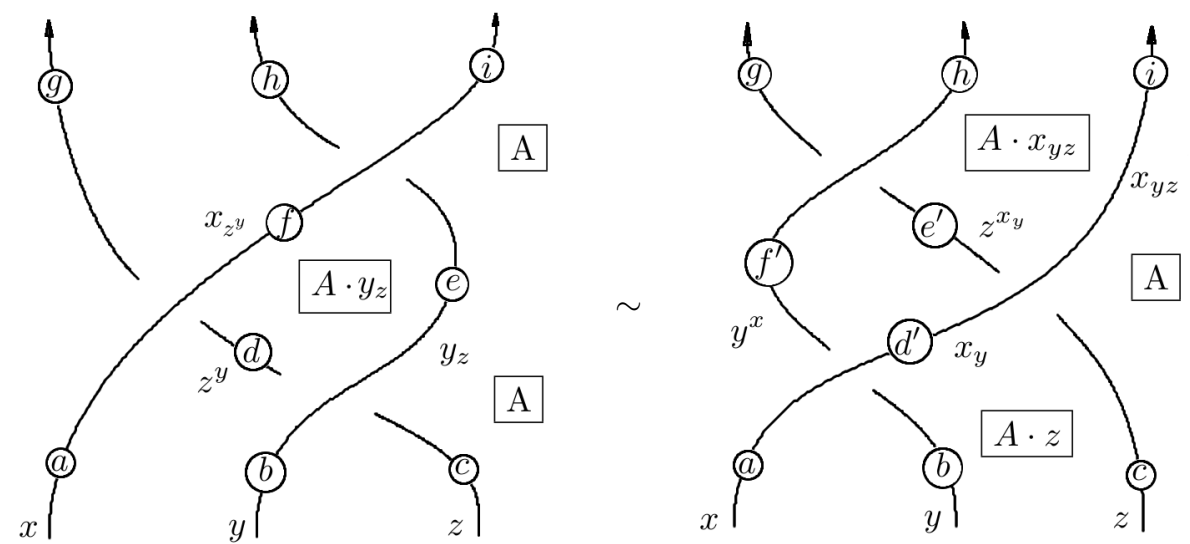

For instance, comparing the coefficients of the bead $i$ before and after the Reidemesiter III moves, we need $r_{A, x_{z}, y_{z}} r_{A \cdot y_{z}, x, z^{y}} i=r_{A, x_{y}, z} r_{A \cdot z, x, y} i$.

Let $X=\left\{x_{1}, \ldots, x_{n}\right\}$ be a finite birack, $S=\left\{A_{1}, \ldots, A_{m}\right\}$ a finite $X$-shadow, and $k$ a ring with identity. We can define an $(X, S)$-module structure on $k$ by selecting $t_{A, x, y}, r_{A, x, y} \in k^{\times}$and $s_{A, x, y} \in k$ such that the ideal in definition 4 is zero; the automorphisms and endomorphisms are then given by left multiplication by the elements $t_{A, x y}, s_{A, x, y}$ and $r_{A, x, y}$. Such a module structure can be conveniently encoded by an $3 n \times k n$ block matrix

$$
M_{R}=\left[\begin{array}{c|c|c}
T_{1} & S_{1} & R_{1} \\
\hline T_{2} & S_{2} & R_{2} \\
\hline \vdots & \vdots & \vdots \\
\hline T_{m} & S_{m} & R_{m}
\end{array}\right]
$$

where the $(i, j)$ entry of $T_{l}$ is $t_{A_{l}, x_{i}, x_{j}}$ and similarly for $S_{l}$ and $R_{l}$.

Example 2 Consider the birack $X$ and $X$-shadow $S$ with matrices

$$
M_{X}=\left[\begin{array}{ll|ll}
1 & 1 & 2 & 2 \\
2 & 2 & 1 & 1
\end{array}\right], \quad M_{X, S}=\left[\begin{array}{ll}
2 & 2 \\
3 & 3 \\
1 & 1
\end{array}\right] .
$$


Our python computations reveal $128 X, S$-module structures on $R=\mathbb{Z}_{3}$ including for instance

$$
M_{M}=\left[\begin{array}{ll|ll|ll}
1 & 1 & 2 & 2 & 2 & 2 \\
1 & 1 & 2 & 2 & 2 & 2 \\
\hline 2 & 1 & 2 & 1 & 1 & 1 \\
2 & 1 & 1 & 2 & 2 & 2 \\
\hline 2 & 1 & 2 & 2 & 2 & 2 \\
2 & 1 & 2 & 2 & 1 & 1
\end{array}\right]
$$

Let $X$ be a finite birack, $S$ an $X$-shadow, $L$ an oriented link of $c$ components, and let $f$ be an $(X, S)$-labeling of a diagram of $L$. Placing beads $a_{1}, \ldots, a_{n}$ on each semiarc in $L$, we get a free $\mathbb{Z}[X, S]$-module generated by the beads. Each crossing gives us two equations; the quotient of this free $\mathbb{Z}[X, S]$-module by the crossing bead relations is then by construction aninvariant of the $(X, S)$ labeling $f$ of $L$ under $(X, S)$-labled framed Reidemeister moves and $N$-phone cord moves, called the fundamental $\mathbb{Z}[X, S]$-module of $f$, denoted $\mathbb{Z}_{X, S}[f]$. We can express $\mathbb{Z}_{X, S}[f]$ with a coefficient matrix of the homogeneous system given by the crossing equations.

Example 3 Let $X, S$ be as in example 2 and consider the labeling of the figure eight knot $4_{1}$ pictured below. We obtain the listed presentation matrix for $\mathbb{Z}_{X, S}[f]$.

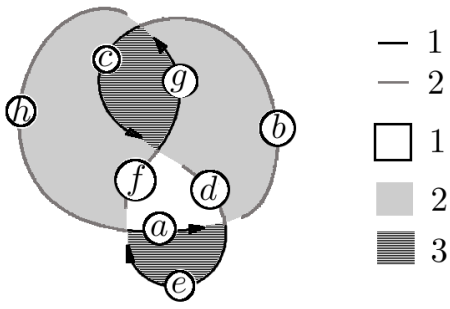

$$
M_{\mathbb{Z}_{X, S}[f]}=\left[\begin{array}{cccccccc}
0 & 0 & 0 & 0 & t_{3,2,1} & -1 & 0 & s_{3,2,1} \\
-1 & 0 & 0 & 0 & 0 & 0 & 0 & r_{3,2,1} \\
t_{3,2,1} & -1 & 0 & s_{3,2,1} & 0 & 0 & 0 & 0 \\
0 & 0 & 0 & r_{3,2,1} & -1 & 0 & 0 & 0 \\
0 & 0 & s_{1,1,2} & 0 & 0 & 0 & -1 & t_{1,1,2} \\
0 & -1 & r_{1,1,2} & 0 & 0 & 0 & 0 & 0 \\
0 & 0 & -1 & t_{1,1,2} & 0 & 0 & s_{1,1,2} & 0 \\
0 & 0 & 0 & 0 & 0 & -1 & r_{1,1,2} & 0
\end{array}\right]
$$

\section{Enhancing the Counting Invariant}

Let $L$ be a blackboard framed oriented link, $X$ a birack of finite rank $N, S$ a finite $X$-shadow and $M$ a $\mathbb{Z}[X, S]$-module.

Definition 5 The shadow module multiset invariant of a link $L$ of $c$ components with respect to the $X, S$-shadow module $M$ is the multiset

$$
\Phi_{X, S, M}^{M}(L)=\left\{\left|\operatorname{Hom}_{\mathbb{Z}[X, S]}\left(\mathbb{Z}_{X, S}[f], M\right)\right|: f \in \mathcal{L}((L, \mathbf{w}),(X, S)), \mathbf{w} \in\left(\mathbb{Z}_{N}\right)^{c}\right\}
$$

where $\mathcal{L}((L, \mathbf{w}),(X, S))$ is the set of shadow labelings of the link diagram $L$ with writhe vector $\mathbf{w}=\left(w_{1}, \ldots, w_{c}\right)$.

The shadow module polynomial invariant is

$$
\Phi_{X, S, M}(L)=\sum_{\mathbf{w} \in\left(\mathbb{Z}_{N}\right)^{c}}\left(\sum_{f \in \mathcal{L}((L, \mathbf{w}),(X, S))} u^{\left|\operatorname{Hom}_{\mathbb{Z}[X, S]}\left(\mathbb{Z}_{X, S}[f], M\right)\right|}\right) .
$$

By construction, for every bead labeling of an $X, S$-labeling of $L$ by beads in $M$, there is a unique corresponding bead labeling of every $X, S$-labeled diagram obtained from $L$ by blackboard framed oriented Reidemeister moves and $N$-phone cord moves. Hence, the set of such $M$-labelings is a signature of the $X, S$-labeling of $L$, and the multiset of these signatures over the set of all $X, S$-labelings forms an enhancement of the birack counting invariant. More formally, we have: 
Theorem 2 If $L$ and $L^{\prime}$ are ambient isotopic classical links, then for any finite birack $X, X$-shadow $S$ and $X, S$-module $M$, we have

$$
\Phi_{X, S, M}^{M}(L)=\Phi_{X, S, M}^{M}\left(L^{\prime}\right) \quad \text { and } \quad \Phi_{X, S, M}(L)=\Phi_{X, S, M}\left(L^{\prime}\right) .
$$

Remark 4 The birack module invariants defined in $\left[3\right.$. coincide with the special case of $\Phi_{X, S}^{M}$ where the shadow $S$ is a singleton set for classical links.

To compute the shadow module invariant of a link $L$ with respect to an $X, S$-module structure $M$ on a commutative ring $G$, we obtain a presentation matrix for $\mathbb{Z}_{X, S}[f]$ for each shadow labeling $f$ of $L$ and replace the $t_{A, x, y}, s_{A, x, y}$ and $r_{A, x, y}$ with their values in $M$. The cardinality $n$ of the solution space of resulting matrix is then the signature of $f$, and $f$ contributes $u^{n}$ to the overall invariant. We then repeat this computation for all shadow labelings over all writhe vectors of $L$.

Example 5 For a simple example, let us compute $\Phi_{X, S}^{M}(K)$ for the trefoil knot $3_{1}$ with respect to the birack, shadow, and $X, S$-module structure on $\mathbb{Z}_{3}$ from example 2 . Since the birack rank of $X$ is $N=2$, we need to consider diagrams of $3_{1}$ with writhes equal to 0 and $1 \bmod 2$. There are six total $X, S$-labelings of an even-writhe diagram of $3_{1}$ and no valid labelings of an odd-writhe diagram. The labeling $f$ below has the listed presentation matrix for $\mathbb{Z}_{X, S}[f]$.

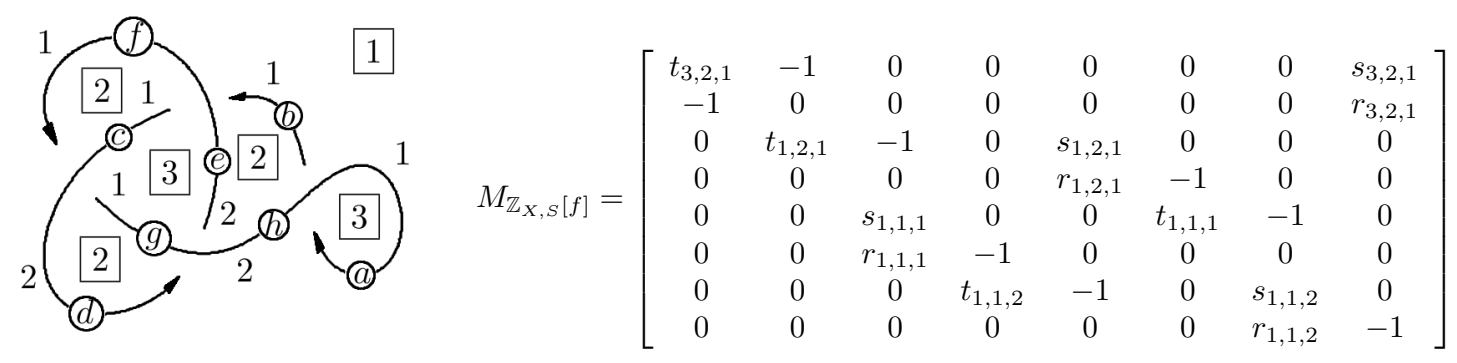

Replacing $t_{A, x, y}, s_{A, x, y}$ and $r_{A, x, y}$ with their values in $R$ and row-reducing over $\mathbb{Z}_{3}$, we have

$$
\left[\begin{array}{llllllll}
2 & 2 & 0 & 0 & 0 & 0 & 0 & 2 \\
2 & 0 & 0 & 0 & 0 & 0 & 0 & 1 \\
0 & 1 & 2 & 0 & 2 & 0 & 0 & 0 \\
0 & 0 & 0 & 0 & 2 & 2 & 0 & 0 \\
0 & 0 & 2 & 0 & 0 & 1 & 2 & 0 \\
0 & 0 & 2 & 2 & 0 & 0 & 0 & 0 \\
0 & 0 & 0 & 1 & 2 & 0 & 2 & 0 \\
0 & 0 & 0 & 0 & 0 & 0 & 2 & 2
\end{array}\right] \rightarrow\left[\begin{array}{llllllll}
1 & 1 & 0 & 0 & 0 & 0 & 0 & 1 \\
0 & 1 & 0 & 0 & 0 & 0 & 0 & 2 \\
0 & 0 & 1 & 0 & 1 & 0 & 0 & 2 \\
0 & 0 & 0 & 1 & 2 & 0 & 0 & 1 \\
0 & 0 & 0 & 0 & 1 & 2 & 2 & 2 \\
0 & 0 & 0 & 0 & 0 & 0 & 1 & 1 \\
0 & 0 & 0 & 0 & 0 & 0 & 0 & 0 \\
0 & 0 & 0 & 0 & 0 & 0 & 0 & 0
\end{array}\right]
$$

Thus, the shadow labeling $f$ contributes $u^{9}$ to $\Phi_{X, S}^{M}\left(3_{1}\right)$. Repeating this for all shadow labelings, we obtain $\Phi_{X, S}^{M}\left(3_{1}\right)=6 u^{9}$; this detects the knottedness of $3_{1}$, since the unknot has $\Phi_{X, S}^{M}$ (Unknot) $=6 u^{3}$. We note that this shows that $\Phi_{X, S}^{M}$ is a properly stronger invariant than the unenhanced shadow counting invariant, since all classical knots have shadow counting invariant value $\Phi_{X, S}^{\mathbb{Z}}=6$.

Example 6 Let $X, S$ and $M$ be the birack, shadow, and $X, S$-module structure on $\mathbb{Z}_{5}$ with matrices

$$
M_{B}=\left[\begin{array}{ll|ll}
1 & 1 & 2 & 2 \\
2 & 2 & 1 & 1
\end{array}\right], \quad M_{s}=\left[\begin{array}{ll}
2 & 2 \\
1 & 1
\end{array}\right], \quad M_{R}=\left[\begin{array}{ll|ll|ll}
1 & 2 & 2 & 2 & 2 & 2 \\
1 & 2 & 2 & 2 & 4 & 4 \\
\hline 1 & 3 & 2 & 1 & 4 & 4 \\
1 & 3 & 4 & 2 & 3 & 3
\end{array}\right]
$$


We computed $\Phi_{X, S}^{R}$ for all prime classical knots with up to eight crossings and all prime classical links with up to seven crossings; these are listed in the table below.

\begin{tabular}{l|l}
$\Phi_{X, S}^{R}$ & $L$ \\
\hline $4 u^{5}$ & $3_{1}, 5_{2}, 6_{1}, 6_{2}, 6_{3}, 7_{1}, 7_{2}, 7_{3}, 7_{5}, 7_{6}, 7_{7}, 8_{1}, 8_{2}, 8_{3}, 8_{4}, 8_{5}, 8_{6}, 8_{7}, 8_{10}, 8_{12}, 8_{13}, 8_{14}, 8_{15}, 8_{17}, 8_{19}, 8_{20}$ \\
$4 u^{25}$ & $4_{1}, 5_{1}, 7_{4}, 8_{8}, 8_{9}, 8_{11}, 8_{16}, 8_{18}, 8_{21}$ \\
$8 u^{5}$ & $L 2 a 1, L 4 a 1, L 5 a 1, L 6 a 1, L 6 a 3, L 7 a 1, L 7 a 3, L 7 a 4, L 7 a 5, L 7 a 6, L 7 n 1, L 7 n 2$ \\
$8 u^{25}$ & $L 6 a 2, L 7 a 2$ \\
$16 u^{5}$ & $L 6 a 4, L 6 a 5, L 6 n 1$ \\
$16 u^{25}$ & $L 7 a 7$
\end{tabular}

Example 7 For our final example, we note that our python computations show that $\Phi_{X, S}^{R}$ is not determined by the Alexander polynomial. The $X, S$-module structure $M$ on $\mathbb{Z}_{3}$ given by

$$
M_{X}=\left[\begin{array}{lll|lll}
1 & 3 & 1 & 3 & 3 & 3 \\
2 & 2 & 2 & 2 & 2 & 2 \\
3 & 1 & 3 & 1 & 1 & 1
\end{array}\right], M_{S}=\left[\begin{array}{lll}
2 & 2 & 2 \\
1 & 1 & 1
\end{array}\right], M_{M}=\left[\begin{array}{lll|lll|lll}
1 & 1 & 1 & 2 & 0 & 1 & 1 & 1 & 1 \\
1 & 1 & 1 & 0 & 0 & 0 & 2 & 1 & 2 \\
1 & 1 & 1 & 1 & 0 & 2 & 1 & 1 & 1 \\
\hline 2 & 2 & 2 & 2 & 0 & 1 & 2 & 2 & 2 \\
2 & 2 & 2 & 0 & 0 & 0 & 1 & 2 & 1 \\
2 & 2 & 2 & 1 & 0 & 2 & 2 & 2 & 2
\end{array}\right]
$$

detects the difference between the Alexander-equivalent knots $8_{18}$ and $9_{24}$ with $\Phi_{X, S}^{R}\left(8_{18}\right)=4 u^{3}+$ $4 u^{27} \neq 4 u^{3}+4 u^{9}=\Phi_{X, S}^{R}\left(9_{24}\right)$. Our python code computed the values of $\Phi_{X, S}^{R}$ for the prime knots with up to eight crossings in the table below.

\begin{tabular}{l|l}
$\Phi_{X, S}^{R}$ & $L$ \\
\hline $4 u^{3}+4 u^{9}$ & $3_{1}, 6_{1}, 7_{4}, 7_{7}, 8_{5}, 8_{10}, 8_{11}, 8_{15}, 8_{19}, 8_{20}, 8_{21}$ \\
$4 u^{3}+4 u^{27}$ & $8_{18}$ \\
$8 u^{3}$ & $4_{1}, 5_{1}, 5_{2}, 6_{2}, 6_{3}, 7_{1}, 7_{2}, 7_{3}, 7_{5}, 7_{6}, 8_{1}, 8_{2}, 8_{3}, 8_{4}, 8_{6}, 8_{7}, 8_{8}, 8_{9}, 8_{12}, 8_{13}, 8_{14}, 8_{16}, 8_{17}$
\end{tabular}

\section{Questions for Future Research}

In this section we collect a few questions for future research.

Quandle and biquandle labelings have been used with 3-cocycles to define invariants of knotted links and surfaces in $R^{4}$. What is the correct generalization of shadow modules to the case of knotted surfaces?

We have defined only the simplest possible invariant using shadow modules, namely counting shadow module labelings. Any quantity computable from a shadow module labeled diagram that is preserved by labeled Reidemeister moves defines and enhancement of the counting invariant. Obvious ideas include rack/birack homology with shadow module coefficients and homology theories with chain groups generated by shadow module elements themselves. What enhancements of the shadow module counting invariant can be found?

Moreover, we have only computed examples of shadow module structures on $\mathbb{Z}_{n}$; shadow modules on finite non-commutative rings such as $\left(\mathbb{Z}_{n}\right)^{m}$ should prove interesting.

We have found examples showing that $\Phi_{X, S}^{M}$ is not determined by the Alexander polynomial. What about other invariants such as twisted Alexander polynomials, Jones and colored Jones polynomials, HOMFLYpt, Khovanov and Knot Floer homologies?

python code for computing the invariants described in this paper is available from the second author's website at http://www.esotericka.org. 


\section{References}

[1] N. Andruskiewitsch and M. Graña. From racks to pointed Hopf algebras. Adv. Math. 178 (2003) $177-243$.

[2] D. Bar-Natan (Ed.). The Knot Atlas. http://katlas.math.toronto.edu/wiki/Main_Page

[3] R. Bauernschmidt and S. Nelson. Birack modules and their link invariants. arXiv:1103.0301

[4] T. Carrell and S. Nelson. On rack polynomials. arXiv:0809.5075, to appear in J. Alg. Appl.

[5] J. S. Carter, M. Elhamdadi, M. Graña and M. Saito. Cocycle knot invariants from quandle modules and generalized quandle homology. Osaka J. Math. 42 (2005) 499-541.

[6] W. Chang and S. Nelson. Rack shadows and their invariants. arXiv:0910.3002, To appear in $J$. Knot Theory Ramifications.

[7] R. Fenn, M. Jordan-Santana and L. Kauffman. Biquandles and virtual links. Topology Appl. 145 (2004) 157-175.

[8] R. Fenn and C. Rourke. Racks and links in codimension two. J. Knot Theory Ramifications 1 (1992) 343-406.

[9] R. Fenn, C. Rourke and B. Sanderson. Trunks and classifying spaces. Appl. Categ. Structures 3 (1995) 321-356.

[10] A. Haas, G. Heckel, S. Nelson, J. Yuen, Q. Zhang. Rack Module Enhancements of Counting Invariants. arXiv:1008.0114, to appear in Osaka J. Math.

[11] D. Joyce. A classifying invariant of knots, the knot quandle. J. Pure Appl. Algebra 23 (1982) 37-65.

[12] L. H. Kauffman and D. Radford. Bi-oriented quantum algebras, and a generalized Alexander polynomial for virtual links. Contemp. Math. 318 (2003) 113-140.

[13] S. V. Matveev. Distributive groupoids in knot theory. Math. USSR, Sb. 47 (1984) 73-83.

[14] S. Nelson. Link invariants from finite racks. arXiv:0808.0029 to appear in Fund. Math.

[15] S. Nelson. Link invariants from finite biracks. arXiv:1002.3842.

Department of Mathematics

Claremont McKenna College

850 Columbia Ave.

Claremont, CA 91711

knots@esotericka.org

Department of Mathematics

Pomona College

610 North College Ave

Claremont, CA 91711 